\title{
Tau LFV and LNV at Belle II
}

\author{
Tomoyuki Konno*t \\ Kitasato University \\ E-mail: tkonno@kitasato-u.ac.jp
}

\begin{abstract}
Charged lepton flavor and number violating $\tau$ lepton decays ( $\tau$ LFV and $\tau$ LNV) are strongly suppressed in the Standard Model (SM) while New Physics scenarios beyond the SM predict branching fractions in the range of $\left(O\left(10^{-7}\right)-O\left(10^{-9}\right)\right)$, which are reachable in the current or near-future experiments. The $\tau$ LFV and LNV modes are studied using almost all data samples from the Belle experiment at the KEKB collider to set $90 \%$ confidence level upper limits of $O\left(10^{-8}\right)$ on the branching fractions. The Belle II experiment started physics data taking in 2019 to achieve 50 times higher statistics of the Belle data in the coming ten years. The physics prospect on the $\tau$ LFV and LNV decay searches in the Belle II experiment, as extrapolations of the Belle results are presented.
\end{abstract}

The 21st international workshop on neutrinos from accelerators (NuFact2019)

August 26 - August 31, 2019

Daegu, Korea

\footnotetext{
* Speaker.

${ }^{\dagger}$ For the Belle II collaboration.
} 


\section{Introduction}

In the Standard Model (SM), charged Lepton flavor violating (cLFV) decay is highly suppressed and not reachable with current experimental techniques, even if neutrino oscillations are taken into account. The branching fractions of $\tau \rightarrow \mu \gamma$ and $\tau \rightarrow \mu \mu \mu$ are expected to be $O\left(10^{-49} \sim\right.$ $10^{-52}$ ) and $O\left(10^{-53} \sim 10^{-56}\right)$ [1], respectively. On the other hand, various scenarios as extensions of the SM, New Physics (NP), naturally allow some cLFV processes. Many NP models, such as SUSY models, predict that several cLFV processes have large branching fractions detectable in current experiments. An observation of the cLFV process is a clear signature of the NP because the SM process is entirely suppressed. The $\tau$ lepton is suitable for cLFV searches because it is the most massive lepton and can decay into various hadrons, providing many decay channels. According to the recent predictions summarized in Table 1, experimental sensitivities to the branching fractions are required to be better than $O\left(10^{-7}\right)$. Comparison among various cLFV decay modes is helpful to determine the NP models since scales of the predicted branching fractions differ among both decay modes and the NP models. In addition to the LFV modes, $\tau$ lepton is predicted to have flavor number violating (cLNV) decay modes $(\tau \rightarrow \ell h h)$ in the NP models. The cLNV modes are also exciting since it has a clear hint to the light NP models.

Table 1: Summary of the predicted branching fractions in the $\tau$ LFV decays among the NP models [2].

\begin{tabular}{|c|c|c|}
\hline Physics models & $B(\tau \rightarrow \mu \gamma)$ & $B(\tau \rightarrow \mu \mu \mu)$ \\
\hline SM $+v$ mixing & $10^{-49} \sim 10^{-52}$ & $10^{-53} \sim 10^{-56}[1]$ \\
SM+heavy Majorana $v_{R}$ & $10^{-9}$ & $10^{-10}$ \\
Non-universal Z' & $10^{-9}$ & $10^{-8}$ \\
SUSY SO(10) & $10^{-8}$ & $10^{-10}$ \\
mSUGRA + seesaw & $10^{-7}$ & $10^{-9}$ \\
SUSY Higgs & $10^{-10}$ & $10^{-7}$ \\
\hline
\end{tabular}

\section{2. cLFV and cLNV at $B$ factories}

$B$ factory is also an $\tau$ factory since the cross section of $\tau^{+} \tau^{-}$pair production is $\sigma_{\tau \tau} \simeq 0.9 \mathrm{nb}^{-1}$, almost the same as $B \bar{B}$ production, $\sigma_{B \bar{B}} \simeq 1 \mathrm{nb}^{-1}$. The KEKB accelerator $[3,4]$ is an $e^{+} e^{-}$asymmetric energy $\left(e^{+}: 3.5 \mathrm{GeV} / e^{-}: 8 \mathrm{GeV}\right)$ collider with the center-of-mass $(\mathrm{CM})$ energy corresponding to the $\Upsilon(4 S)$ resonance. A data sample corresponding to an integrated luminosity of $\approx 1 \mathrm{ab}^{-1}$ from the Belle experiment, the world-highest luminosity, has been accumulated by the end of the operation in 2010; this sample includes $\sim 10^{9} \tau^{+} \tau^{-}$pairs, which can be used to search for the $\tau$ LFV decay modes.

All analyses that search for $\tau$ cLFV decay are based on the tagging method of $\tau^{+} \tau^{-}$pairs. One $\tau$, as the signal side, decays into a LFV mode while the other $\tau$, as the tag side, decays into one (or three) charged particles in the SM process followed by any number of photons and neutrinos. The event selection is tuned based on particle identification and kinematic information for each of the LFV decay separately, to maximize its sensitivity. Missing momentum due to neutrinos 
provides significant information to reduce the SM $\tau^{+} \tau^{-}$background, since $\tau$ LFV modes have neither neutrino nor missing momentum. Finally, we either observe an LFV $\tau$ decay or set an upper limit by comparing the expected and observed numbers of events in the signal regions. In the remainder of this section, a few channels studied by Belle are reviewed.

Decays of the $\tau$ to three charged leptons are the cleanest search modes and an almost background free analysis is possible in the Belle experiment, due to the three lepton tracks on the signal side. Six decay modes are studied using $782 \mathrm{fb}^{-1}$ of data [5]. No signal event is observed in all decay modes, while the backgrounds are expected to be less than 0.21 events. No evidence is observed, and the $90 \%$ confidence level (C.L.) upper limits on the branching fractions are set in the range of $(1.5-2.7) \times 10^{-8}$.

The channel $\tau \rightarrow \ell \gamma$ is most interesting since the branching fraction in new physics models is induced by one-loop diagrams with heavy particles. On the other hand, there are some difficulties in separating the signals from backgrounds because only two particles are in the signal side, and many background sources such as $\mu$ pair with initial-state radiation can easily mimic these signals. $4.5 \times 10^{-8}$ and $1.2 \times 10^{-8}$ at $90 \%$ C.L. are set for $\tau \rightarrow \mu \gamma$ and $\tau \rightarrow e \gamma$, respectively [6].

The decay $\tau^{-} \rightarrow \ell^{-} h^{+} h^{\prime-}$ violates only the lepton flavor, while $\tau^{-} \rightarrow \ell^{+} h^{-} h^{\prime-}$ violates both the lepton flavor and the lepton number (LNV). Eight modes of $\tau^{-} \rightarrow \ell^{-} h^{+} h^{\prime-}$ and six modes of $\tau^{-} \rightarrow \ell^{+} h^{-} h^{-}$are studied. The lepton number conserving modes are expected to be enhanced in the Higgs-mediated models. On the other hand, the searches for LNV decays are motivated by the Majorana neutrino model. A data sample corresponding to an integrated luminosity of $854 \mathrm{fb}^{-1}$ was analyzed to search for these modes [7]. Main backgrounds are coming from the pion misidentification into muon of $\tau->\pi \pi \pi v$ decay, and into electron of $\tau->\pi^{0} \pi v$ decay. The 90 $\%$ C.L. upper limits on the branching fractions are set in the range of $(2.0-8.4) \times 10^{-8}$.

\section{Status of the Belle II}

The Belle II experiment [8] performed its Phase II commissioning from April to July of 2018. The Phase II data sample corresponds to an integrated luminosity of $500 \mathrm{pb}^{-1}$ at the $\Upsilon(4 S)$ energy. Belle II started Phase III of data taking with an almost complete vertex detector.

The tau pairs are reconstructed using a Phase II data sample of $291 \mathrm{pb}^{-1}$ by looking for 3-1 prong events. Reconstructed tracks provide event topology variables, such as thrust vector, which is used to select tau pair candidates. After selection, 9800 events remain as candidates. Figure 1 shows the invariant-mass distribution of the three charged pions coming from $\tau \rightarrow 3 \pi \nu$ candidates, which is consistent with Monte Carlo (MC) simulations. A measurement of $\tau$ lepton mass is carried out using the Phase II data as the first trial in Belle II, based on the method introduced by the ARGUS collaboration. In $\tau \rightarrow 3 \pi v$ events, a pseudomass $M_{\min }$ is obtained with the following definition:

$$
M_{\min }=\sqrt{M_{3 \pi}^{2}+2\left(E_{\text {beam }}-E_{3 \pi}\right)\left(E_{3 \pi}-P_{3 \pi}\right)},
$$

where $E_{\text {beam }}$ is the beam energy and $M_{3 \pi}, E_{3 \pi}, P_{3 \pi}$ are the invariant mass, the energy, and the momentum of the three pions in CMS, respectively. An empirical probability density function (p.d.f.) is used to estimate the $\tau$ lepton mass:

$$
F\left(M_{\min } ; a, b, c, m^{*}\right)=\left(a \times M_{\min }+b\right) \times \tan ^{-1}\left(\frac{m^{*}-M_{\min }}{c}\right)+P_{1}\left(M_{\min }\right),
$$


where $a, b$, and $c$ are real values and the parameter $m^{*}$ is an estimator of the $\tau$ lepton mass. A fit of the p.d.f. in the pseudomass region from 1.70 to $1.85 \mathrm{GeV} / c^{2}$, shown in Figure 2, obtains $m_{\tau}=(1776.4 \pm 4.8$ (stat) $) \mathrm{MeV} / c^{2}$, which is in good agreement with the previous measurements [9].

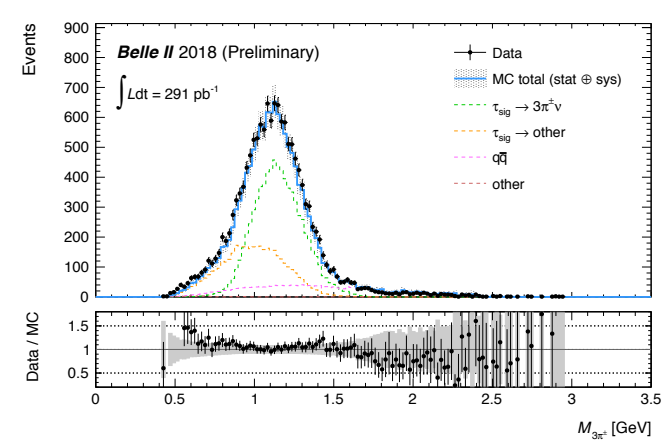

Figure 1: Invariant-mass distribution of the three pions coming from $\tau \rightarrow 3 \pi v$ candidates reconstructed in Phase II data [10].

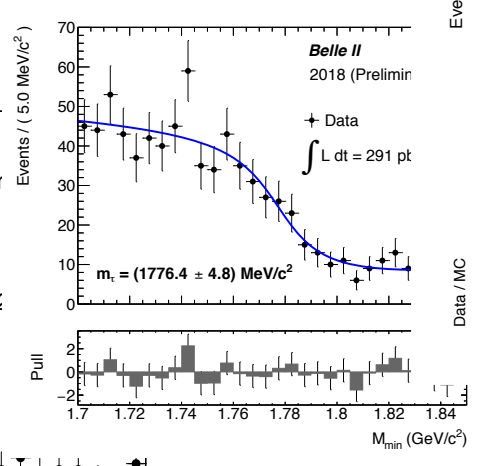

Figure 2: Distribution of pseudomass of $\tau \rightarrow 3 \pi v$ candidates reconstructed using the Phase II data. the blue line is the fit results of the function 3.2 .

\section{Future Prospects}

As for prospects of $\tau$ LFV in the Belle II experiment, the sensitivities for $\tau$ LFV decay modes depend on the contamination of the remaining backgrounds. The sensitivity for $\tau \rightarrow \mu \gamma$ is scaled proportionally to of $1 / \sqrt{\mathscr{L}}$, where $\mathscr{L}$ is the integrated luminosity, while the sensitivity for $\tau \rightarrow$ $\mu \mu \mu$ can be scaled linearly since the remaining background is negligible. The upper limits of the branching fractions of $\tau \rightarrow \mu \gamma$ and $\tau \rightarrow \mu \mu \mu$ are estimated to be $O\left(10^{-8} \sim 10^{-9}\right)$ and $O\left(10^{-9} \sim\right.$ $\left.10^{-10}\right)$, respectively. Comparisons of the upper limits on the branching fractions from the current experiments (CLEO, BaBar and Belle) with extrapolations for the Belle II and the LHCb upgrade are summarized in Fig. 3. In all of the decay modes, the upper limits can be improved 10 times compared to the Belle results using $50 \mathrm{ab}^{-1}$ of the Belle II data sample. These limits reach the predicted branching fractions in some of the NP models.

\section{Summary}

Various $\tau$ LFV decays are studied for using about $1 \mathrm{ab}^{-1}$ of data collected by the Belle detector at the KEKB asymmetric $e^{+} e^{-}$collider, resulting in upper limits on the branching fractions of the $\tau$ LFV decay modes of $O\left(10^{-7} \sim 10^{-8}\right)$, while several NP models predict large branching fractions to be a bit lower than the current upper limits by $O\left(10^{-2} \sim 10^{-3}\right)$. The sensitivities to the $\tau$ LFV decay modes are expected to be improved by a factor of 10 by using the 50 times higher statistics from the Belle II experiment to reach the NP predictions. 


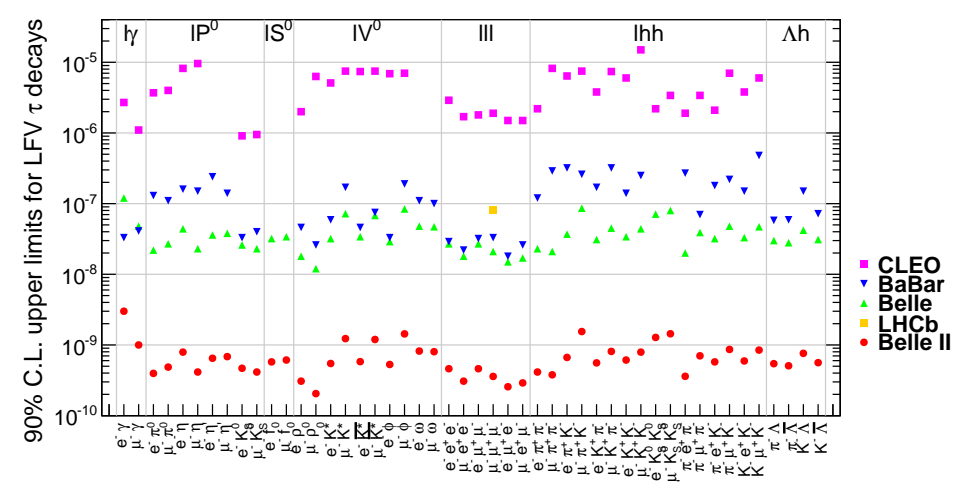

Figure 3: $90 \%$ C.L. upper limits on the branching fractions of cLFV decay modes based on results from CLEO (pink), BaBar (blue) and Belle (green), and extrapolations for Belle II $\left(50 \mathrm{ab}^{-1}\right)$ and LHCb upgrade $\left(50 \mathrm{fb}^{-1}\right.$, yellow) [11].

\section{Acknowledgments}

This work was supported by Grant-in-Aid for Scientific Research(A) JSPS KAKENHI Grant Numbers JP19H00682.

\section{References}

[1] G. Hernandez-Tome et al., Flavor violating leptonic decays of $\tau$ and $\mu$ leptons in the Standard Model with massive neutrinos, Eur. Phys. J. C 79-84 (2019).

[2] M. Blanke, et al., Charged Lepton Flavour Violation and $(g-2)_{\mu}$ in the Littlest Higgs Model with T-Parity: a clear Distinction from Supersymmetry, JHEP 0705, 013 (2007).

[3] S. Kurokawa et al., Overview of the KEKB accelerators, Nucl. Instrum. Methods A 499, 1 (2003).

[4] A. Abashian et al., (Belle Collaboration), The Belle Detector, Nucl. Instrum. Methods A 479, 117 (2002).

[5] K.Hayasaka, et al., (Belle collaboration), Search for Lepton Flavor Violating Tau Decays into Three Leptons with 719 Million Produced Tau+Tau-Pairs, Phys. Lett. B 687, 139-143 (2010).

[6] K.Hayasaka, et al., (Belle collaboration), New search for $\tau \rightarrow \mu \gamma$ and $\tau \rightarrow$ e $\gamma$ decays at Belle, Phys. Lett. B 16, 666 (2008).

[7] Y. Miyazaki et al., (Belle Collaboration), Search for lepton-flavor and lepton-number-violating decay modes, Phys. Rev. D 57, 3873 (1998), Phys. Lett. B 719, 346 (2013).

[8] T. Abe et al., Belle II Technical Design Report, KEK Report 2010-1 [physics.ins-det/1011.0352].

[9] M. Tanabashi et al., (Particle Data Group), Review of Particle Physics, Phys. Rev. D, 98, 030001 (2018).

[10] D. Rodríguez Pérez, (on behalf of Belle II Collaboration), Prospects for $\tau$ lepton physics at Belle II, proceedings in 17th Conference on Flavor Physics and CP Violation (FPCP 2019), [hep-ex/1906.08950].

[11] E. Kou et al., The Belle II Physics Book, PTEP, 12, 123 C01 (2019) [hep-ex/1808.10567]. 